\title{
Study on the Lane Mark Identification Method in Intelligent Vehicle Navigation
}

\author{
Gaoying Zhi \& Wencheng Guo \\ College of Computer and Automatization \\ Tianjin Polytechnic University \\ Tianjin 300160, China \\ E-mail:cong_107@163.com
}

\begin{abstract}
The intelligent vehicle navigation based on machine vision is the important part to realize Intelligent Transportation System (ITS), and it includes road detection, obstacle detection and motion control. Combining with foreign and domestic latest research trends, in this article, we mainly study the typical method of road detection, and point out the research and development tendency of intelligent vehicle navigation of technology based on the machine vision.
\end{abstract}

Keywords: Intelligent vehicle, Machine vision, Road detection, Intelligent Transportation System (ITS)

\section{Introduction}

When human being enters into the 21st century, with the development of the transportation industry, the problems of transportation become more and more serious, for example, the vehicles on roads are crowd, the traffic accidents are frequent, the traffic environment is deteriorated, the energy sources are intense, and the environmental pollution is continually serious. To solve these problems, it must be restricted from many aspects only depending on the extension of road establishment construction. Under this situation, in order to systematically solve the problems of road traffic, we must completely consider vehicles and road, utilize all kinds of high and new technologies, and the new scientific research and application area, i.e. Intelligent Transportation System (ITS). ITS integratively applies advanced information processing technology, computer technology, data traffic technology, sensor technology, electric control technology, GPS technology, automatic control theory, operational research, artificial intelligence and other high and new technologies and theories to transportation, service control and vehicle intelligent. The intelligent vehicle navigation based on machine vision is the important part of ITS, and it mainly completes tasks of road detection and obstacle detection and controls vehicles safely running on roads. Because the computer vision system possesses many characters such as cheap price, multiple purposes and simple structure, and conveniently integrating with other sensors, it has broad application foreground and become the main direction of present research, and the real-time, robustness and reliability are key targets to test the intelligent vehicle navigation system. In this article, we mainly study the road identification in the intelligent vehicle navigation and summarize the research and development tendency of intelligent vehicle navigation of technology based on the machine vision.

\section{Road detection}

In the intelligent vehicle navigation based on machine vision, the road detection is mainly to estimate the position and direction of vehicle on the road and control vehicles safely running on the road. Otherwise, it can confirm search range for the next obstacle detection, reduce the range of obstacle detection, and reduce the arithmetic complexity and error identification rate. Because the roads in practice differ in thousands ways, the road detection is a very complex problem about pattern recognition, but there is no universal arithmetic of road detection at present. To reduce the treatment difficulty of the road detection and the detection speed of arithmetic, the proper introduction of basic hypothesis about road will help to simplify the problems. In fact, the present road detection systems are to design corresponding detection arithmetic aiming at all kinds of concrete roads. The road supposes in common use at present includes following aspects. The first one is the hypothesis of road form, and the road simplifying model is a sort of effective method, and it implements the road diction by the familiar road form. The second one is the hypothesis of road width, and if the road width can be fixed or changed slowly, the problem of road detection can be simplified to be the parallel road character detection problem. The third one is the consistent hypothesis of road surface character, and usually the area of corresponding road surface in the image possesses the consistent character differing to non-road area, such as the 
character of gray level, the character of color and the character of texture, so the method of clustering can be adopted to detect the road area. The fourth one is the hypothesis of road evenness, and when the system obtains the image characters (such as the lane mark line), it needs transform the characters from the image coordinate system to the vehicle coordinate system in order to control the vehicle, and if the forward road is even, so we can utilize the known vidicon positioning information to implement transformation. The fifth one is the hypothesis of special interest area, and though the operation quantity of real-time treatment of road following is very large, but according to the physical restriction and the continuity restriction, it an be simplified through the time pertinence between neighbor frames, and we can only analyze and look for expected characters in some interest areas, but not analyze the whole image, and accordingly reduce the treatment time and reduce the complexity of the arithmetic. The present road detection arithmetic mainly includes two sorts, the method based on road character and the method based on road model.

\subsection{Road detection based on characters}

The road detection arithmetic based on character mainly includes two parts, the character abstraction and the character integration. First analyze the road image and confirm which characters should be selected, then use these characters to implement image partition, and finally compose the partition results according to certain rules to visual road expression. The road character selection can be considered respectively from area view and edge view. The character selection based on area is mainly to analyze the differences between road area and non-road area, and the both dissimilarity can be characters such as color, texture and gray level. In the colorful road image, the road color has large difference with the environment, and we can use this character to realize the partition of road area. Because of uneven illumination and shadow, the colors on the road in one image can not go all the way, and the environmental colors differ in thousands ways, and only the partitions of road area and non-road area are difficult to realize the road partition. In SCARF, the road area and the non-road area are divided into four sorts to enhance the nicety of the partition. Some articles used the method of morphologic to realize the local clustering of similar areas in the image and complete the area integration by means of the rules and obtain the complete road area. The texture is a distinct character in the road image. The road texture is relatively single and the arrangement is ordered, but the texture of the environment is disorderly and unsystematic. We can realize the road area partition according to this character. The edge of the road is the focus noticed by human all along. In usual arithmetic, we first get the edge grads in the image, then follow the edges with big grades according to the grads direction and finally obtain the whole boundary. Because of the discontinuity of the boundary, the key is to obtain the description of the whole roadside by means of desultory edge combination which is usually realized by the fitting method, and the fitting methods include the whole line fitting, the subsection linear fitting and other sorts. Under strong illumination, the influence of shadow can not be ignored, and some times the edge of shadow is stronger than the road edge, so the elimination of shadow edge is a difficulty. Usually we can utilize the scrambling of shadow edge and the color character of shadow area to solve this problem. Because various characters have their own advantages and disadvantages, the adoption of various characters such as edge and area at the same time is a sort of tendency. In KRUSL4, it used the edge information, area information and road experience knowledge to make the road detection arithmetic more exact and reliable. To reduce the computation of the arithmetic, we usually use the Kalman filter to predict the possible position of the road in next frame of the image and reduce the treatment area of the image and quicken the speed of road detection.

\subsection{Road detection based on models}

Both the structured road and the non-structured road have relatively regular road mark or road edge, and the establishment of corresponding curve model according to the road edge form is another difficulty in the road detection. The simplest model is the line model. In the finitely long range, the roadsides are supposed to be two parallel lines. In the forward looking image, the roadsides are two radials intersected on the vanishing point. First, we find the line existing in the image though the Hough transformation method or other methods, then we abstract the parallel lines from the image. We can also implement the reverse perspective transformation, and transform the forward looking image to the quasi-planform, and then abstract the parallel lines from the image. The parabola and multinomial model is a sort of familiar arc road description. The parameter confirmation of this model is very important. To describe border road structure, some articles used the B-spline curve model, because the B-spline curve can form curves with random forms through a group of control points. First, confirm the initial position of B-spline rhough the vanishing point, and then detect the control point of B-spline model in the whole image by means of the minimum mean energy method. This model can effectively restrain the effects of road surface stain, shadow and uneven illumination, but the complexity of arithmetic is very high. Comparing with the road detection method based on character, the road detection based on model can effectively overcome some influences of environmental factors such as road surface stain, uneven illumination and shadow. But when the road fall short of the beforehand hypothesis, the model will be not effective, so the selection of the model is very important. To enhance the robustness of the road model, we usually use the present detection results to timely update the parameters of the road model, and make the road model more accord with the description of the actual road. 


\section{Tendencies of research and development}

From above analysis, the road tracking based on machine vision has obtained large achievements. Most experiment vehicles can basically realize independent running in most time. The research emphasis of this domain has gone to the stage of system performance enhancement and stride forwards the practicality and production from the stage of exploring experiment. In the process of road detection, the road image collected suffers following factors such as the differences of construction time, construction mode and construction material of various road sections, changes of environment, the changes of the weather, the changes of illumination, and lane line deformity induced by water stain and smirch, which also induce certain difficulties for the real-time and exact road detection. At present, the road following with high reliability under all-weather and multiple situations will the aim to be further studied in the future. Most systems use the visible light vidicon at present, which can only work in the day, and can not work at night, but the nights is the frequent periods of traffic accident, so the intelligent vehicle navigation is most needed. The IR thermal imaging technology can be used to realize the vehicle navigation at night, which is a hot spot in the present researches. However, because its price is very expensive and the imaging quality cannot be satisfied, there is a long way to achieve the practicality. The following technology of the structured road has been basically matured, and the attention begins to turn to the complex urban traffic environment with low speed and backland wild environment, and try to realize the continual and independent work under multiple road situations. In the urban traffic environment, the traffic mark and lane mark detection is one new hot spot in the research of intelligent vehicle navigation. The reliability is the aim of the road following system, which is the key to realize the practicality and production of the system. To enhance the reliability, more and more systems begin to adopt the integrated technology including multiple sensors integration for hardware and multiple algorithms integration for software. Except for visual sensor, the laser range finder, millimeter wave radar and some initiative range equipments are used together to enhance the reliability of the detection result. For the algorithm, many sorts of detection algorithms are used at the same time to decide the final detection results by the methods of voting or blur ratiocination.

\section{References}

Guan, Yanping, Heyue, Liu, Peizhi \& Lvlin. (2005). Lane and Road Detection Based on Colorful Image. Journal of Computer Applications. No.25(12). p.2931-2934.

Hu, Minghao, Yang, Wenjie, Ren, Mingwu \& Yang, Jingyu. (2005). Vision-based Road Detection Algorithm. Computer Engineering and Design. No.26(7). p.1704-1706.

Ji, Tianming, Heyue, Yutong \& Wang, Shaojun. (2005). Real-time Lane Detection for Intelligent Vehicle Navigation System. Journal of Computer Applications. No.25(B12). p.228-230, 232.

Li, Minqi \& Zhouquan. (2006). A Kind of Road Detection from Remote Sensing Images. Modern Electronics Technique. No.29(6). p.125-127.

Li, Shuxiao \& Chang, Hongxing. (2007). Novel Road Detection and Tracking Algorithm for Aerial Images. Journal of Beijing University of Aeronautics and Astronautics. No.33(4). p.445-449.

Lu, Weina \& Shi, Zhongke. (2007). Synchronous Detection of the Lane Marking and Road Boundary on Monocular Vision. Chinese Journal of Sensors and Actuators. No.20(5). p.1171-1175.

Pi, Yanni, Shi, Zhongke \& Huangjin. (2005). A Lane and Preceding Vehicle Detection System Based on Monocular Vision. Computer Engineering. No.31(23). p.186-188.

Wangjing \& Wangqiang. (2007). Segmented Self-adaptive Road Detection Technique Based on Algorithm Integration. Computer Measurement \& Control. No.15(4). p.455-456, 459.

Wangqiang, Wang, Youren \& Wang, Yongming. (2006). Self-adaptive Road Detection Based on Road Scene Matching and Reconfigurable Hardware Technique. Transducer and Microsystem Technologies. No.25(1). p.69-72.

Wang, Yongming, Wang, Youren, Yaorui \& Zhangzhai. (2006). Road Detection Method Based on Algorithm Fusion and Reconfigurable Hardware. Opto-electronic Engineering. No.33(2). p.132-137.

$\mathrm{Xu}$, Quansheng, Yumiao \& Zhou, Haihua. (2006). Method of Realizing Road Detection in Advanced Vehicle Control System. Journal of Shenyang University of Technology. No.28(2). p.150-152, 173.

Yangming, Lu, Jianye, Wanghong \& Zhangbo. (2001). Vision Based Road Following. Pattern Recognition and Artificial Intelligence. No.14(2). p.186-193.

Yang, Wenjie, Hu, Minghao \& Yang, Jingyu. (2006). A Fast Edge Based Unstructured Road Detection Algorithm. Computer Science. No.33(5). p.257-260.

Zhangwei, Huang, Xiyue \& Yang, Shanggang. (2006). Lane Detection in Navigation System of Vehicle. Journal of Chongqing University (Natural Science Edition). No.29(8). p.87-90. 